\title{
Correction to: Engaging a product-focused sales force in solution selling: interplay of individual- and organizational-level conditions
}

\section{Anna Salonen $^{1}$ (I) $\cdot$ Harri Terho ${ }^{1} \cdot$ Eva Böhm $^{2} \cdot$ Ari Virtanen $^{3} \cdot$ Risto Rajala $^{3}$}

Published online: 2 July 2020

(C) Academy of Marketing Science 2020

Correction to: Journal of the Academy of Marketing Science.

https://doi.org/10.1007/s11747-020-00729-Z

The original article was published online with incorrect Table 3 layout. The correct table is shown below:

The original article has been corrected.

$\triangle$ Anna Salonen

anna.k.salonen@utu.fi

1 University of Turku, Turku, Finland

2 TU Dortmund University, Dortmund, Germany

3 Aalto University, Espoo, Finland 
Table 3 Respondent Demographics

SALESPEOPLE

\begin{tabular}{lll}
\hline Sales Experience & N & Percent \\
\hline $\mathbf{5}<$ & 45 & 24.5 \\
$\mathbf{5 - 9}$ & 37 & 20.1 \\
$\mathbf{1 0 - 1 4}$ & 39 & 21.2 \\
$\mathbf{1 5 - 1 9}$ & 24 & 13 \\
$\mathbf{2 0 - 2 4}$ & 23 & 12.5 \\
$\mathbf{2 5}>$ & 16 & 8.7 \\
\hline Total & 184 & 100
\end{tabular}

\begin{tabular}{lll}
\hline Gender & N & Percent \\
\hline Male & 167 & 90.8 \\
Female & 10 & 5.4 \\
\hline Missing & 7 & 3.8 \\
Total & 184 & 100
\end{tabular}

\begin{tabular}{lll}
\hline Education level & N & Percent \\
\hline Master or higher & 52 & 28.3 \\
Bachelor & 56 & 30.4 \\
Vocational degree & 36 & 19.6 \\
High school & 26 & 14.1 \\
Other & 13 & 7.1 \\
\hline Missing & 1 & .5 \\
Total & 184 & 100
\end{tabular}

\begin{tabular}{lll}
\hline Education type & N & Percent \\
\hline Technical degree & 85 & 46.2 \\
Business degree & 50 & 27.2 \\
Other & 33 & 17.9 \\
Missing & 16 & 8.7 \\
\hline Total & 184 & 100
\end{tabular}

\begin{tabular}{lll}
\hline Age & N & Percent \\
\hline $\mathbf{2 5}<$ & 1 & .5 \\
$\mathbf{2 5}-\mathbf{2 9}$ & 10 & 5.4 \\
$\mathbf{3 0 - 3 4}$ & 24 & 13 \\
$\mathbf{3 5 - 3 9}$ & 37 & 20.1 \\
$\mathbf{4 0 - 4 4}$ & 36 & 19.6 \\
$\mathbf{4 5 - 4 9}$ & 25 & 13.6 \\
$\mathbf{5 0 - 5 4}$ & 24 & 13 \\
$\mathbf{5 5 - 5 9}$ & 16 & 8.7 \\
$\mathbf{6 0}>$ & 11 & 6 \\
\hline Total & 184 & 100
\end{tabular}

CHAMPIONS

\begin{tabular}{lll}
\hline Sales Experience & N & Percent \\
\hline $\mathbf{5}<$ & 8 & 34.8 \\
$\mathbf{5 - 9}$ & 4 & 17.4 \\
$\mathbf{1 0 - 1 4}$ & 4 & 17.4 \\
$\mathbf{1 5 - 1 9}$ & 5 & 21.7 \\
$20-24$ & 0 & 0 \\
$\mathbf{2 5}>$ & 0 & 0 \\
\hline Missing & 2 & 8.7 \\
Total & 23 & 100
\end{tabular}

\begin{tabular}{lll}
\hline Gender & N & Percent \\
\hline Male & 21 & 91.3 \\
Female & 1 & 4.3 \\
\hline Missing & 1 & 4.3 \\
Total & 23 & 100
\end{tabular}

\begin{tabular}{lll}
\hline Education level & N & Percent
\end{tabular}

\begin{tabular}{lll}
\hline Master or higher & 7 & 30.4
\end{tabular}

Bachelor $\quad 10 \quad 43.5$

$\begin{array}{lll}\text { Vocational degree } & 6 & 26.1\end{array}$

High school $\quad 0 \quad 0$

Other

$\begin{array}{ll}0 & 0\end{array}$

Total

$23 \quad 100$

\begin{tabular}{lll}
\hline Education type & N & Percent \\
\hline Technical degree & 13 & 56.5 \\
Business degree & 5 & 21.7 \\
Other & 4 & 17.4 \\
Missing & 1 & 4.3 \\
\hline Total & 23 & 100
\end{tabular}

\begin{tabular}{lll}
\hline Age & N & Percent \\
\hline $\mathbf{2 5}<$ & 0 & 0 \\
$\mathbf{2 5}-\mathbf{2 9}$ & 0 & 0 \\
$\mathbf{3 0 - 3 4}$ & 4 & 17.4 \\
$\mathbf{3 5 - 3 9}$ & 3 & 13 \\
$\mathbf{4 0 - 4 4}$ & 6 & 26.1 \\
$\mathbf{4 5 - 4 9}$ & 3 & 13 \\
$\mathbf{5 0 - 5 4}$ & 3 & 13 \\
$\mathbf{5 5 - 5 9}$ & 3 & 13 \\
$\mathbf{6 0}>$ & 1 & 4.3 \\
\hline Total & 23 & 100
\end{tabular}

MANAGERS

\begin{tabular}{lll}
\hline Sales Experience & N & Percent \\
\hline $\mathbf{5}<$ & 5 & 19.2 \\
$\mathbf{5 - 9}$ & 5 & 19.2 \\
$\mathbf{1 0 - 1 4}$ & 2 & 7.7 \\
$\mathbf{1 5 - 1 9}$ & 4 & 15.4 \\
$\mathbf{2 0 - 2 4}$ & 2 & 7.7 \\
$\mathbf{2 5}>$ & 4 & 15.4 \\
\hline Missing & 4 & 15.4 \\
Total & 26 & 100
\end{tabular}

\begin{tabular}{lll}
\hline Gender & $\mathrm{N}$ & Percent \\
\hline Male & 23 & 88.5 \\
Female & 1 & 3.8 \\
\hline Missing & 2 & 7.7 \\
Total & 26 & 100
\end{tabular}

\begin{tabular}{lll}
\hline Education level & N & Percent \\
\hline Master or higher & 11 & 42.3 \\
Bachelor & 11 & 42.3 \\
Vocational degree & 3 & 11.5 \\
High school & 1 & 3.8 \\
Other & 0 & 0 \\
\hline Total & 26 & 100
\end{tabular}

\begin{tabular}{lll}
\hline Education type & N & Percent \\
\hline Technical degree & 12 & 46.2 \\
Business degree & 10 & 38.5 \\
Other & 2 & 7.7 \\
Missing & 2 & 7.7 \\
\hline Total & 26 & 100
\end{tabular}

\begin{tabular}{lll}
\hline Age & N & Percent \\
\hline $\mathbf{2 5}<$ & 0 & 0 \\
$\mathbf{2 5 - 2 9}$ & 0 & 0 \\
$\mathbf{3 0 - 3 4}$ & 0 & 0 \\
$\mathbf{3 5 - 3 9}$ & 2 & 7.7 \\
$\mathbf{4 0 - 4 4}$ & 2 & 7.7 \\
$\mathbf{4 5 - 4 9}$ & 8 & 30.8 \\
$\mathbf{5 0 - 5 4}$ & 7 & 26.9 \\
$\mathbf{5 5 - 5 9}$ & 4 & 15.4 \\
$\mathbf{6 0}>$ & 1 & 3.8 \\
\hline Missing & 2 & 7.7 \\
Total & 26 & 100
\end{tabular}

Publisher's note Springer Nature remains neutral with regard to jurisdictional claims in published maps and institutional affiliations. 